\title{
HRTEM Study of Au-Cu Alloy Nano Particles: Concentration Dependence
}

\author{
NAIR Dilip Kumar Saha, Kenji Koga and Harutoshi Takeo
}

HRTEM observation was performed on the as-grown and annealed clusters of Au-13, 25, 39 and 57 at.\% $\mathrm{Cu}$ alloys. In the as-grown alloy clusters, it was difficult to understand their structures clearly, because most of the clusters were found in irregular shapes without showing clear structural patterns, although several clusters showed distorted icosahedral (Ic) or Ic-like structures. Almost $85 \%$ of the observed structures were seen either complex (C) or face-centered cubic (Fcc) and the rest $15 \%$ structures were seen Ic-like but mostly distorted form of it for $\mathrm{Au}-13 \mathrm{at} . \% \mathrm{Cu}$ alloys. In case of $\mathrm{Au}-25$ at. $\% \mathrm{Cu}$ alloy, similar trends but higher rate, 30\%, of Ic structures was observed. In case of $\mathrm{Au}-39$ and 57 at.\% $\mathrm{Cu}$ alloys, very few Ic structures were observed in the as-grown state where about 10 and $6 \%$ of the observed clusters were identified Ic-like, respectively.

Contrary to the as-grown clusters, sharp and very clear Ic structures were observed in the annealed clusters. Almost $30 \%$ of the observed structures were seen Ic patterns with in the size range of 2-6 nm in diameter for Au-13 at.\% Cu alloy clusters. In Au-25 at.\% Cu alloy clusters, we have observed almost $80 \%$ Ic structures with in the size range of $2-6 \mathrm{~nm}$ in diameter and the rest $20 \%$ structures were found to be Fcc and complex. In case of Au-39 and 57 at.\% $\mathrm{Cu}$ alloys, we have observed around 30 and $12 \%$ icosahedral structures, respectively and the rest of the structures show Fcc and complex structures with multiple domain Fcc in the size range of 2-6 nm. All the results are shown in Table 1. Above the size of $6 \mathrm{~nm}$ in diameter, clusters were found to be Fcc structures, either single-domain, multi-domain or distorted structures, indicating bulk nature. We have observed no Ic structures above the size of $6 \mathrm{~nm}$ in diameter in any of the four different $\mathrm{Au}-\mathrm{Cu}$ alloy clusters. Decahedral structure is totally absent in both the as-grown and annealed states.

Table 1. The formation rate of different structures with in the size range of $2-6 \mathrm{~nm}$ in diameter.

\begin{tabular}{lllll}
\hline Alloy composition & \multicolumn{2}{l}{ As-grown clusters (\%) } & \multicolumn{2}{l}{ Annealed clusters (\%) } \\
& \multicolumn{1}{l}{} & & \\
\hline $\mathrm{Au}-13$ at.\% $\mathrm{Cu}$ & Ic (like) & 15 & Ic & 30 \\
& Fcc+C & 85 & Fcc+C & 70 \\
$\mathrm{Au}-25$ at.\% $\mathrm{Cu}$ & Ic (like) & 30 & Ic & 80 \\
& Fcc+C & 70 & Fcc+C & 20 \\
$\mathrm{Au}-39$ at.\% $\mathrm{Cu}$ & Ic (like) & 10 & Ic & 30 \\
& Fcc+C & 90 & Fcc+C & 70 \\
$\mathrm{Au}-57$ at.\% $\mathrm{Cu}$ & Ic (like) & 6 & Ic & 12 \\
& Fcc+C & 94 & Fcc+C & 88 \\
\hline
\end{tabular}

Our main aim was to find out the stable Ic structure's formation rate and the critical size for each of the four different alloy compositions of $\mathrm{Au}-\mathrm{Cu}$ alloy system. The formation rate of Ic was found to be completely different for each alloys and it is concluded that $\mathrm{Au}-25$ at.\% $\mathrm{Cu}$ alloy is the unique and only alloy which can form maximum number of stable Ic structures in the $\mathrm{Au}-\mathrm{Cu}$ alloy system. 